\title{
Main Pharmacological Strategies Against SARS-CoV-2
}

Thais Gonçalves Fontes', Afrânio Côgo Destefani’

\begin{abstract}
There are currently no specific drugs for COVID-19. However, several drugs approved for other situations, as well as several investigative agents, are being studied for the treatment of COVID-19 in several hundred clinical trials worldwide. In anticipation of the results of clinical trials, different medical actors around the world have used drugs empirically and with unknown safety profiles. It should be noted that emerging data demonstrated that cardiovascular comorbidities are very common in patients with COVID-19 and that these patients are at increased risk of death. To trace a path of light through the cloudiness that we live on COVID 19, we conducted a bibliographic search, aiming, mostly, to present concise, relevant and scientific information. The main groups of drugs and their adverse effects and drug interactions were raised. Specific concerns in patients with COVID-19 include underlying structural heart disease, cardiac injury, kidney and liver dysfunction, limited resources for cardiac monitoring and drug interaction. Clear administration protocols must be in place in all hospitals and clinics that use drugs for the treatment of COVID-19, to assist in research and analysis of possible drugs capable of inhibiting the virus without consequent complications.
\end{abstract}

Keywords: COVID-19, SARS-Cov-2, strategy.

\section{INTRODUCTION}

2019- $\mathrm{nCoV}$ belongs to Betacoronavirus, which also contains SARS-CoV and the Middle East Respiratory Syndrome CoV (MERS-CoV) virus. Various drugs have been used in patients, although the effectiveness of some drugs remains controversial ${ }^{1}$. It is known that the estimated incubation period for COVID-19 is up to approximately 14 days from the time of exposure, with an average incubation period of 4 to 5 days. The spectrum of the disease can vary from asymptomatic, mild symptomatic to severe symptomatic - condition with severe pneumonia infection with acute respiratory distress syndrome (ARDS) and death. In a report of 1.482 hospitalized patients with confirmed COVID-19 in the United States, the most common symptoms were coughing (86\%), fever or chills (85\%), shortness of breath (80\%), diarrhea (27\%) and nausea (24\%). Other reported symptoms included, but were not limited to, sputum production, headache, dizziness, rhinorrhea, anosmia, dysgeusia, sore throat, abdominal pain, anorexia and vomiting ${ }^{2}$.

There are currently no drugs approved by the Food and Drug Administration (FDA) for COVID-19. However, several drugs approved for other situations, as well as several investigative agents, are being studied for the treatment of COVID-19 in several hundred clinical trials worldwide ${ }^{2}$. It is important to emphasize that, unfortunately, there is still no elucidated and effective mechanism for this condition ${ }^{3}$. While largescale studies are being conducted in multiple countries, their preliminary results on effective therapies are at

\footnotetext{
${ }^{1}$ Faculty of Biomedical Sciences, Espírito Santo, Cariacica, Brasil
}

Corresponding author.

Afrânio Côgo Destefani, Rua Bolivar de Abreu, 48, Campo Grande, Cariacica/ES, CEP. 29.146-330.

E-mail: afraniocd@gmail.com 
least a few months ahead. Awaiting the results of clinical trials, suppliers around the world are using drugs empirically and with unknown safety profiles. It should be noted that emerging data demonstrated that cardiovascular comorbidities are very common in patients with COVID-19 and that these patients are at increased risk of death ${ }^{4}$.

To trace a path of light through the cloudiness that we live over COVID-19, we conducted a bibliographic search in PubMed, aiming, mostly, to present concise, relevant and scientific information. For this purpose, we performed a prior descriptor check on the Mesh platform, so that the research had a unique language and guaranteed reproducibility: SARS virus, drugs, antiviral agents. 474 articles were found initially and, except of works of explicit nature of bibliographic review, we obtained 217 articles. Consequently, we have restricted to works published in the last 10 years including Homo sapiens, resulting in 11 articles.

\section{RESULTS AND DISCUSSION}

Studies have shown that pharmaceutical products that inhibited coronavirus included neurotransmitter inhibitors, estrogen receptor antagonists, kinase signaling inhibitors, lipid or sterol metabolism inhibitors, protein processing inhibitors and DNA synthesis / repair inhibitors. Regarding antiparasitic or antibacterials, the function was not linked to coronavirus or viruses in general, but they did have antiviral activity against SARS-CoV and MERS-CoV. Interestingly, classes of drugs that appear to inhibit only SARSCoV or MERS-CoV, but not both, have been discovered. Although only a small number of SARS-CoV inhibitors have been identified, they are mainly antiinflammatory drugs, which interfere with the cellular signal associated with the immune response to virus infection. MERS-CoV was specifically blocked by ion transport inhibitors, cytoskeleton (specifically tubulin) and by apoptosis ${ }^{5}$.

In studies, tyrosine kinase inhibitors showed high antiviral potential5. Imatinib mesylate and dasatinib inhibited MERS-CoV and SARS-CoV, while nilotinib inhibited only SARS-CoV. However, a clinical trial study showed that its antiviral action would be dose-dependent. When administered at the time of infection, there was a significant result of inhibition of viral production. However, when administered post-infection, there was no inhibition even though there is a certain action under SARS-VOC replication ${ }^{6}$. In cyto- toxic analysis, imatinib mesylate and dasatinib inhibited low toxicity SARS-CoV and MERS-CoV 5 . Thus, SARS-CoV is more sensitive to both inhibitors, since imatinib mesylate and dasatinib are known inhibitors of the homologous 1 route of Abelson murine leukemia (ABL1) viral oncogene 1 . The ABL1 pathway is a signaling pathway involved in cell differentiation, cell adhesion and response to cell stress. However, overactivation of the ABL1 pathway can lead to chronic myeloid leukemia. Imatinib mesylate and dasatinib have been developed and approved as inhibitors of this route for the treatment of human cancers, including chronic myeloid leukemia. These data suggest that the ABL1 pathway may be important for the replication of many different virus families and, therefore, inhibitors of this pathway have the potential to be broad-spectrum antivirals. Imatinib mesylate and dasatinib inhibit SARS$\mathrm{CoV}$ and MERS-CoV with micromolar EC50s and low toxicity. SARS-CoV appears to be more sensitive to the two ABL1 inhibitors 5 .

\section{HCV AND HIV INHIBITORS IN SARS TEST}

The drugs examined include HIV protease inhibitors used primarily in combination with other antiviral drugs to treat adults and children. Also, other antiviral drugs have shown activity against $\mathrm{HCV}$ and good oral bioavailability. In protein analysis of the virus, the drug-receptor fit showed that 5 of the 13 protease inhibitors, which are reported to have a high score in thermodynamic terms of protein-drug complex, could be selected in the hall of new SARS-specific antiviral drugs. Promising results have also been seen for $\mathrm{CoV}-2$ protease inhibitors. The SARS-CoV-2 spike protein (or protein $\mathrm{S}$ ) is present in the envelope as a homotrimer, as in the case of other enveloped viruses. Inhibitors are typically designed to interact with the top of the protein, interfering with the host membrane's binding to the protein's fusogenic activity. Fit of three different inhibitors was performed on the protein spike ${ }^{7}$. Simeprevinir, on the other hand, showed surprising results because a similarity between the main $\mathrm{HCV}$ protease and the SARS-CoV-2 counterpart was identified. However, the two proteases share a topology very similar to the activation site. It is also important to note that Enfuvirtide presented an objection in the position of fitting the Spike protein, making the connection and transition difficult ${ }^{7}$. The pharmacodynamics of HIV protease inhibitors raises concerns about the adequacy 
of drug levels to inhibit SARS-CoV-2 protease can be achieved with oral dosing. Besides, lopinavir / ritonavir was evaluated in a small randomized controlled study in patients with COVID-19 with results that did not show efficacy. In a clinical trial that randomized 199 patients with lopinavir $400 \mathrm{mg}$ / ritonavir $100 \mathrm{mg}$ orally twice daily for 14 days or according to the standard of care (SOC), patients randomized to lopinavir / ritonavir did not have less time in regarding clinical improvement. There was a lower, but not statistically significant, mortality rate for the lopinavir / ritonavir group (19.2\%) than in the SOC group (25.0\%) and shorter ICU stay for patients in the lopinavir / ritonavir group than in the SOC group (6 days vs. 11 days; difference $=-5$ days; $95 \% \mathrm{CI},-9$ to 0$)^{7}$.

\section{REMDESIVIR}

The clinical isolate of COVID-19 in vitro was used to evaluate antivirals. Remdesivir had a positive and expected response in the post-viral entry stage, consistent with its antiviral effectiveness against Ebola. Because these compounds were used in human patients with a safety history and are effective against various diseases, the study suggests that they be evaluated in human patients suffering from the new coronavirus disease ${ }^{1}$. In a randomized study, Remdesivir was used in an infected group. In these, serum creatinine and aminotransferase values were higher in the ZMapp and Remdesivir groups than in the MAb114 and REGN-EB3 groups; patients in the latter groups had better results, despite similar durations in the course of the disease ${ }^{8}$.

\section{CHLOROQUINE AND HYDROXYCHLOROQUINE}

Chloroquine is widely distributed throughout the body, including the lung, after oral administration. In addition to its antiviral activity, chloroquine has an immunomodulatory activity that can synergistically increase its antiviral effect in vivo ${ }^{1}$. Based on the results of physiological-based pharmacokinetic models (PBPK), the study found that cells in vitro showed that both chloroquine and hydroxychloroquine have good antiviral activity and decrease viral replication in a concentration-dependent manner'. Hydroxychloroquine exhibited a superior in vitro antiviral effect compared to chloroquine when the drug was added before the viral challenge. It was also observed that, with longer incubation times, the EC50 values for chloroquine and hydroxychloroquine tended to decrease. The inhibitory effect of chloroquine was weak: this observation was carried out using EC50 values of hydroxychloroquine always lower than the EC50 values of chloroquine, indicating that hydroxychloroquine has a more potent antiviral activity ${ }^{9}$. In addition, the association of the two drugs was also evaluated, but without good results. Research has highlighted that they recognize the combination of the two drugs, and that it confers a potential risk of prolonging the QTc interval and requires daily monitoring of electrocardiogram patients ${ }^{10}$.

\section{DEXAMETHASONE}

Favorable modulation of the immune response is considered one of the possible mechanisms by which corticosteroids can be beneficial in the treatment of severe infections by respiratory coronavirus, including $\mathrm{CO}$ VID-19, SARS and MERS. Common cases to severe cases of these infections are the presence of hypercytokinaemia and the development of acute lung injury or acute respiratory distress syndrome (ARDS) ${ }^{11}$. Pathologically, diffuse alveolar damage is found in patients who die from these infections. An increasing volume of clinical trial data from patients with severe community-acquired pneumonia, ARDS and septic shock suggest benefits of low to moderate dose of corticosteroids about mortality and length of stay ${ }^{11}$.

\section{FLAVONOIDS}

Flavonoids are an important type of natural products. In particular, they belong to a type of secondary plant metabolites with a polyphenolic structure widely found in fruits and vegetables. Interestingly, some flavonoids also have antiviral activity ${ }^{12}$. In a study conducted with a flavonoid library, specifically a flavonol (herbacetin) and two flavones (rhoifolin and pectolinarin), they have been reported to inhibit the proteolytic activity of SARS-CoV 3CLpro ${ }^{12}$. Widely distributed in the plant kingdom and the most abundant in the world of the flavonoid family, quercetin was shown to be more effective in another study presenting selective inhibition of the activity against SCV NTPase / helicase. On the other hand, due to the cytotoxicity associated with the test compounds, only five derivatives of quercetin showed selective antiviral activity in $\mathrm{HCV}$ replication cells $^{13}$. This impasse was also observed by another study, highlighting the mutational fusion in the presence of the antiviral inhibitors flavonoids, flavipiravir and oseltamivir ${ }^{14}$. The test result indicates important conclusions: flavonoids initially have a wide range of binding 
affinity to SARS-CoV 3CLpro due to their hydrophobic aromatic rings and hydrophilic hydroxyl groups; second, the presence of groups of carbohydrates severely influences the affinity and mode of binding of the chromen-4-one fraction. Based on the result, the strategy for dealing with SARS targeting the SARS-CoV 3CLpro can be designed recently. An additional study is underway to make derivatives that lead to better inhibitory compounds based on this study ${ }^{12}$.

\section{ADVERSE EVENTS}

\section{Imatinibe}

Imatinib has relatively low cardiotoxicity, while second and third generation TKIs (dasatinib, nilotinib, bosutinib and ponatinib) have been reported to have multiple vascular adverse effects, including vascular occlusive events, peripheral arterial occlusive disease, QT prolongation and hypertension pulmonary. In murine models fed with tyrosine, it was found that the accumulation of tyrosine in the vascular wall correlates with insulin-induced endothelial relaxation, interfering with the production of nitric oxide. Patients with chronic kidney disease and type 2 diabetes have an increased tyrosine load, which can contribute to the development of vascular complications. Patients at high risk for cardiovascular complications and profound MRI may benefit from a TKI dose reduction or suspension program. However, CML cases without comorbidities did not have significant changes ${ }^{15}$.

\section{Remdesivir}

Remdesivir is not FDA approved, but is available through an emergency use authorization for the treatment of hospitalized adults and children with COVID-19 and is currently being investigated in clinical trials. In a randomized, placebo-controlled study, it was found that the 28-day mortality rate was similar in both branches of the study. There was no difference between groups in SARS-CoV-2 viral load at baseline and the rate of decline over time was similar between the two groups. The number of participants who had adverse events was similar in both groups $(66 \%$ and $64 \%$ of participants in the remdesivir and placebo groups, respectively). More participants in the remdesivir group than the placebo node discontinued therapy due to adverse events ( $12 \%$ vs. $5 \%$ of participants in the remdesivir and placebo groups, respectively). However, the study ended early. As a result, the sample size did not have enough power to detect differences in clinical results. In addition, it is known that this drug can cause gastrointestinal symptoms (e.g., nausea, vomiting), elevated levels of transaminase, and elevated prothrombin time (without changing the international normalized ratio). Concentration can be affected by strong CYP inducers, but the interaction is not expected to be clinically significant ${ }^{2}$.

\section{Chloroquine and Hydroxychloroquine}

Concerns about cardiovascular toxicity are associated, particularly because of its known relationship with cardio electric instability, characterized by prolongation of the QT interval (the time required for ventricular depolarization and repolarization). Under specific conditions, after depolarization, they can trigger ventricular arrhythmias. This propensity to the occurrence of arrhythmia is more frequently observed in individuals with structural cardiovascular diseases. There is great concern in the use of these drugs because heart disease and cardiac injury have been reported with high frequency during COVID-194. Reports have documented severe dysrhythmias in patients with COVID-19 treated with chloroquine or hydroxychloroquine, often in combination with azithromycin and other drugs that prolong the QTc interval. Given the risk of dysrhythmias, FDA warns against the use of chloroquine or hydroxychloroquine in the treatment of COVID- 19 out of a hospital or clinical trial2. In a preliminary safety outcome of a randomized, doubleblind, phase IIb clinical study (CloroCovid-19 Study), to evaluated the safety and efficacy of two different dosages of $\mathrm{QC}$ as adjunctive therapy for patients hospitalized with SARS- CoV-2, the CQ arm showed QTc> $500 \mathrm{~ms}(25 \%)$ and a tendency to higher lethality (17\%) than the lowest dose in the first 81 recruited patients. Preliminary findings suggest that the highest dose of QC (10-day regimen) should not be recommended for the treatment of COVID-19 because of its potential safety risks. As a result, the researchers prematurely stopped recruiting patients for this follow-up ${ }^{3}$.

\section{HIV protease inhibitors}

The duration of hospital admissions and the time to release viral RNA from samples from the respiratory tract did not differ between the lopinavir/ritonavir and SOC arms. Nausea, vomiting and diarrhea were all more frequent in the lopinavir/ritonavir group. The study was not blind, which may have affected assessments of clinical improvement. The study was insufficient to show small effects ${ }^{2}$. 


\section{Flavonoids}

Exposure to increased levels of flavonoids, either through diet or supplementation, can potentially overload the body, leading to the formation of reactive oxygen species and, ultimately, DNA damage. In addition, these effects can be increased in fetal development, where there is rapid cell growth, which can increase sensitivity to phytochemical exposure. Little is known about the toxicology of excessive flavonoid intake, while the beneficial attributes are overemphasized ${ }^{16}$.

\section{Dexametasone}

In trials with low to moderate doses of corticosteroids, the main adverse effect has been hyperglycemia ${ }^{11}$. A systematic review of trials with corticosteroids (mainly at low doses) taking into account the occurrence of severe sepsis and septic shock, did not identify an increased risk of gastroduodenal bleeding, superinfection or neuromuscular weakness; an association with an increased risk of hyperglycemia and hypernatremia was noted. Dexamethasone has minimal mineralocorticoid activity and practically does not affect the sodium and water balance, avoiding possible problems with fluids. In pregnancy, prednisolone $40 \mathrm{mg}$ taken orally (or intravenous hydrocortisone $80 \mathrm{mg}$ twice daily) should be used instead of dexamethasone ${ }^{11}$.

\section{References}

1. Wang, M. et al. Remdesivir and chloroquine effectively inhibit the recently emerged novel coronavirus (2019-nCoV) in vitro. Cell Res. 30, 269-271 (2020).

2. NIH Guidelines. Coronavirus Disease 2019 (COVID-19) Treatment Guidelines. 2019, (2020).

3. Şimşek Yavuz, S. \& Ünal, S. Antiviral treatment of covid-19. Turkish J. Med. Sci. 50, 611-619 (2020).

4. Naksuk, N., Lazar, S. \& Peeraphatdit, T. B. Cardiac safety of offlabel COVID-19 drug therapy : a review and proposed monitoring protocol. 1-7 (2020) doi:10.1177/2048872620922784.

5. Dyall, J. et al. Repurposing of clinically developed drugs for treatment of Middle East respiratory syndrome coronavirus infection. Antimicrob. Agents Chemother. 58, 4885-4893 (2014).

6. Coleman, C. M. et al. Abelson Kinase Inhibitors Are Potent Inhibitors of Severe Acute Respiratory Syndrome Coronavirus and Middle East Respiratory Syndrome Coronavirus Fusion. J. Virol. 90, 8924-8933 (2016).

7. Calligari, P., Bobone, S., Ricci, G. \& Bocedi, A. Molecular Investigation of SARS - CoV-2 Proteins and. (2020).

8. Mulangu, S. et al. A randomized, controlled trial of Ebola virus disease therapeutics. N. Engl. J. Med. 381, 2293-2303 (2019).

9. Yao, X. et al. In Vitro Antiviral Activity and Projection of Optimized Dosing Design of Hydroxychloroquine for the Treatment of Severe Acute Respiratory Syndrome Main point : Hydroxychloroquine was found to be more potent than chloroquine at inhibiting SARS-CoV-2 in vit. Clin. Infect. Dis. 2, 1-25 (2020).

\section{CONCLUSION}

The administration of antiviral candidate drugs soon after the onset of symptoms can reduce transmissibility to other individuals, thereby decreasing viral excretion in the respiratory secretions of patients infected with SARS-CoV-2 - which usually peak at 5 to 6 days after the onset of symptoms and lasts up to 14 days. Specific concerns in patients with COVID-19 include underlying structural heart disease, cardiac injury, kidney and liver dysfunction, limited resource for cardiac monitoring and also drug interactions. Clear administration protocols must be in place in all hospitals and clinics that use these drugs for the treatment of COVID-19, given the possible adverse events that can worsen the clinical picture. Knowing the pharmacological principles of each drug and its management will assist in research and analysis of possible chemical substances capable of inhibiting the virus without subsequent complications for the patient.

Compliance with ethics requirements: The authors declare no conflict of interest regarding this article. The authors declare that all the procedures and experiments of this study respect the ethical standards in the Helsinki Declaration of 1975, as revised in 2008(5), as well as the national law. Informed consent was obtained from all the patients included in the study.

10. Chowdhury, M. S., Rathod, J. \& Gernsheimer, J. A Rapid Systematic Review of Clinical Trials Utilizing Chloroquine and Hydroxychloroquine as a Treatment for COVID-19. Acad. Emerg. Med. (2020) doi:10.1111/acem.14005.

11. EudraCT. Randomised Evaluation of Covid-19 Therapy (Recovery). Recovery 1-22 (2020).

12. Jo, S., Kim, S., Shin, D. H. \& Kim, M. S. Inhibition of SARS-CoV 3CL protease by flavonoids. J. Enzyme Inhib. Med. Chem. 35, 145-151 (2020).

13. Park, H. R., Yoon, H., Kim, M. K., Lee, S. D. \& Chong, Y. Synthesis and antiviral evaluation of 7-0-arylmethylquercetin derivatives against SARS-associated coronavirus (SCV) and hepatitis C virus (HCV). Arch. Pharm. Res. 35, 77-85 (2012).

14. Jensen, J. D. \& Lynch, M. Considering mutational meltdown as a potential SARS-CoV-2 treatment strategy. Heredity (Edinb). (2020) doi:10.1038/s41437-020-0314-z.

15. Caocci, G. et al. Metabolomic Analysis of Patients with Chronic Myeloid Leukemia and Cardiovascular Adverse Events after Treatment with Tyrosine Kinase Inhibitors. J. Clin. Med. 9, 1180 (2020)

16. Health, P., Of, I. \& Flavonoid, E. Lars Ernster Commemorative Issue Guest Editors: Enrique Cadenas and Kelvin J . A . Davies. 29, 375-383 (2000). 
